\title{
Geradores Betavoltaicos para Sistemas Eletrônicos Portáteis
}

\author{
Ferreira GS $\uparrow$ and Toufen DL $\dagger$ \\ †Instituto Federal de Educação, Ciência e Tecnologia de São Paulo, IFSP.
}

Resumo. As fontes de energia convencionais, baseadas em processos eletroquímicos, se tornaram um elemento cotidiano, sendo empregadas em inúmeros equipamentos eletrônicos, como smartphones e notebooks. Entretanto, mesmo com avanços na tecnologia na última década, estas apresentam uma evolução inferior à esperada, causando uma limitação dos dispositivos eletrônicos, criando uma necessidade por fontes energéticas mais potentes. $\mathrm{O}$ desenvolvimento de um gerador de radioisótopos, baseado em sistemas betavoltaicos, que utilizam processos nãotérmicos gerando energia elétrica com base na emissão de radiação beta, uma tecnologia que permitiria que um eletrônico funcionem por longos períodos, sem necessidade de recarga ou reposições. Entretanto, apesar das vantagens que esta tecnologia nos apresenta, temos o desafio referente aos riscos à saúde ocasionados a exposição indevida a radiação, onde a Comissão Nacional de Energia Nuclear (CNEN), a qual estabelece limites de dosagem, que devem ser seguidos para se projetar de um gerador betavoltaico utilizando materiais e técnicas, permitindo que tais dispositivos sejam desenvolvidos para o manuseio e utilização de forma segura, tendo-se como base o nível de radiação beta emitida e o comportamento do radioisótopo empregado, permitindo o dimensionamento de uma blindagem eficiente e segura.

Palavras-Chave. Gerador, Betavoltaico, Radioisótopo, Dose Radioativa, Blindagem. 


\title{
Betavoltaic Generators for Portable Electronic Systems
}

\author{
Ferreira GS $\uparrow$ Toufen DL $\uparrow$ \\ †Instituto Federal de Educação, Ciência e Tecnologia de São Paulo, IFSP.
}

\begin{abstract}
Conventional energy sources, based on electrochemical processes, have become an everyday element, being used in countless electronic equipment, such as smartphones and notebooks. However, even with advances in technology in the last decade, they show a lower evolution than expected, causing a limitation of electronic devices, creating a need for more powerful energy sources. The development of a radioisotope generator, based on betavoltaic systems, which uses non-thermal processes generating electrical energy based on the emission of beta radiation, a technology that would allow an electronic device to function for long periods, without the need for recharging or replacement. However, despite the advantages that this technology presents us, we have the challenge regarding health risks caused by undue exposure to radiation, where the Comissão Nacional de Energia Nuclear (CNEN), which establishes dosage limits, which must be followed to design of a betavoltaic generator using materials and techniques, allowing such devices to be developed for safe handling and use, based on the level of beta radiation emitted and the behavior of the radioisotope employed, allowing the dimensioning of an efficient shield and safe.
\end{abstract}

Keywords. Generator, Betavoltaic, Radioisotope, Radioactive Dose, Shielding.

Introdução. O físico italiano Alessandro Volta, em 1800, construiu e descreveu a primeira bateria eletroquímica, conhecida como "pilha voltaica". Era constituída empilhando chapas de cobre e zinco, separadas por discos de papel embebidos em salmoura e podia produzir uma 
corrente constante por um período considerável de tempo. A principio, Volta não entendeu que a origem da voltagem, que era devida a reação química, pensando assim que suas células eram uma fonte inesgotável de energia e que os efeitos de corrosão associados aos eletrodos eram um mero incômodo, e não uma conseqüência inevitável de sua operação, como Michael Faraday mostrou em 1834.

O termo "bateria" foi usado para descrever dispositivos elétricos pela primeira vez por Benjamin Franklin, que em 1748, descreveu múltiplos frascos de Leyden, uma espécie de gerador eletrostático, criando uma analogia devido a semelhança com bateria de canhões. Embora as primeiras baterias fossem de grande valor para fins experimentais, na prática, sua voltagem oscilavam e não conseguiam fornecer uma corrente estável por um intervalo de tempo considerável.

Em 1836, John Frederic Daniell, químico britânico, desenvolveu o que ficou conhecida como a Célula Daniells, que consistia de um pote de cobre preenchido com uma solução de sulfato de cobre, na qual estava imerso um recipiente de barro não vidrado cheio de ácido sulfúrico e um eletrodo de zinco. A célula de Daniells foi a primeira fonte prática de eletricidade, tornando-se um padrão da indústria e adotada como uma fonte de energia para redes de telégrafo elétrico. Devido a forma como estas pilhas são constituídas, usando eletrólitos líquidos, se tornam propensas a vazamentos, muitos usavam frascos de vidro para segurar seus componentes, tornando-as frágeis e potencialmente perigosas. Devido a essas características, as células úmidas se tornaram impróprias para uso em aparelhos portáteis, e no começo do século XIX, foram desenvolvidas baterias de células secas, que substituíram o eletrólito líquido por uma pasta, tornou práticos os dispositivos elétricos portáteis.

Em 1913, o físico inglês Henry Moseley (1887-1915), após experimentos envolvendo partículas beta, desenvolveu o que é considerada a primeira bateria atômica do mundo, utilizando o isótopo radioativo do elemento Rádio $(\mathrm{Ra})$ como emissor beta voltaico. 
Em 1977, a NASA, escolheu o projeto de geradores de radioisótopos, baseado em isótopos de Urânio-238, para suprir energia as duas sondas sondas do programa Voyager. Pela fato das sondas terem como jornadas que as distanciariam do Sol, o uso de painéis solares, comuns fonte de energia em satélites e sondas não seria uma escolha viável. Atualmente, 41 anos após o lançamento das sondas, ambas continuam em funcionamento, com alguns sistemas desligados, mas enviando sinais conforme a missão segue, apesar de suas fontes de energia já estejam a cerca de $70 \%$ da potência inicial, considerando a meia-vida de 87.74 anos, do Urânio238.

Geradores Betavoltaicos para Sistemas Eletrônicos Portáteis. O desenvolvimento de baterias betavoltaicas de tamanho reduzido e potencial energético para utilização em dispositivos eletrônicos em geral é uma possibilidade viável, pois os benefícios desta tecnologia, quando comparados a atual tecnologia de baterias, apresenta vantagens notáveis.

O primeiro aspecto a se considerar seria a disponibilidade energética que os dispositivos móveis vem solicitando de acordo com o desenvolvimento tecnológico. Uma bateria convencional é desenvolvida para ter uma alimentação contínua média de 12-16 horas e uma vida útil máximo de 05 anos, podendo variar de acordo com a utilização pelo dispositivo. Uma bateria convencional apresenta vida útil referente a quantificação de energia eletroquímica que é transformada em energia elétrica. O processo coloca o elemento Lítio como um dos mais empregados na construção das baterias atuais, onde sua obtenção natural e posterior processamento, até sua forma metálica, o torna um produto caro e faz com que sua reciclagem também se estabeleça de forma viável.

Uma bateria de radioisótopos poderia ser desenvolvida para fornecer uma quantidade de potência estável, durante um intervalo de tempo consideravelmente longo, pois o processo se manter enquanto ocorrer a reação de decaimento ocorrer, apresenta uma vida útil proporcional á 
razão entre a meia-vida do radioisótopo, utilizado para a emissão das partículas beta, pela sua perda de potência ao decorrer dos anos, A potência apresentaria uma queda relacionada a partir do tempo de vida útil, que é relacionado para que metade do material se deteriore em um período de tempo específico, onde o trítio, isótopo do elemento hidrogênio, apresenta um vida útil de cerca de 12.33 anos e o estrôncio 90, subproduto de reatores nucleares, apresenta meia vida de 29,78 anos.

Atualmente, com a evolução da tecnologia dos materiais, é viável a miniaturização de um gerador de radioisótopos, com base em decaimento beta, processo conhecido como betavoltaico. O decaimento beta é, basicamente, um processo nuclear, onde núcleos atômicos instáveis, buscando estabilidade, acabam emitindo elétrons, ou pósitrons, com alta energia, como resultado dessas alterações no núcleo. Com base na tecnologia e com os materiais atuais, é possível projetar e desenvolver uma célula betavoltaica, de dimensões próximas a uma bateria moderna de um smartphone, que tenha uma potência equivalente ou maior, que não necessite de ciclos de recargas e acima de tudo, respeitando todos os limites de segurança para que os usuários destes aparelhos possam conviver a longo prazo com esses dispositivos.

O primeiro aspecto seria a necessidade energética que os dispositivos móveis vem solicitando de acordo com o desenvolvimento tecnológico. A atual tecnologia de baterias não se desenvolveu ao mesmo passo que a tecnologia empregada nos dispositivos eletrônicos, fazendo com que a fonte de energia acabe que contendo o potencial do dispositivo, pois este não consegue fornecer todo o potencial que o mesmo alcançaria e quando o faz, é por um curto período de tempo.

Metodologia. O princípio de funcionamento de um gerador betavoltaico é relativamente simples. A utilização de partículas beta do radioisótopo para estimular outro elemento metálico, criando uma diferença de potencial entre eles. O radioisótopo, devido ao decaimento e emissão beta, 
acaba apresentando um caráter positivo, enquanto que o elemento estimulado pela radiação beta apresenta um caráter negativo. Esta forma de polarização gera uma diferença de potencial nos elementos participantes e permite que energia seja retirada deste sistema.

Importante citar que além do processo de geração de energia, temos também o processo de contenção do radioisótopo. A radiação beta é baseada na emissão de partículas fundamentais, de alta energia, originado nas reações nucleares. Esta forma de radiação apresenta uma capacidade de penetração considerável e pode apresentar sérios problemas quando interagem com tecidos vivos. Atualmente, temos a disposição materiais que podem facilmente conter radiação beta sem apresentarem problemas, permitindo que o gerador seja seguro e tenha dimensões relativamente pequenas.

Um gerador betavoltaico apresenta desempenho relacionado, principalmente, ao radioisótopo empregado, com duas propriedades relacionadas a ele, seu tempo de meia-vida e a atividade nuclear.

A Atividade Nuclear é relacionada a quantidade de reações de decaimento radioativo que ocorrem no período de 01 segundo. Este é medido em Becquerels (Bq), onde cada radioisótopo apresenta uma atividade distinta. O tempo de meia-vida é o tempo em que o elemento radioativo leva pra que metade de sua massa decaia. Este processo em si pode durar de alguns segundos até milhares de anos.

Entre estas duas propriedades, um radioisótopo ideal para esta aplicação deve apresentar um equilíbrio entre a atividade nuclear com um tempo de meia-vida. Elementos com uma meiavida muito curta liberam muita energia em um curto espaço de tempo, entretanto, caso a meiavida seja longa, ele libera pouca energia durante um longo período.

Outro fator indireto, mas que se destaca, é a forma como o radioisótopo pode ser obtido. Alguns radioisótopos podem ser obtidos de forma natural, encontrados na crosta terrestre, como o Césio-137, ou na forma de subprodutos de outros radioisótopos mais pesados, gerados na serie 
de decaimentos que ele passa até forma um isótopo estável, como o Estrôncio-90, ou de forma artificial, sendo produzidos por técnicas de ativação de núcleos, como o "bombardeamento de nêutrons", onde um elemento pode ser ativado afetando sua estabilidade nuclear e tornando-se radioativo, como ocorre com o Trítio, que pode ser obtido com o bombardeamento de elementos como Lítio ou Boro.

A seguir, temos uma tabela de radioisótopos, com suas devidas propriedades e cálculos relacionando a potência teórica do gerador baseado nele. Todos os cálculos de atividade foram realizados considerando $1 \mathrm{~mol}$, ou $6,022 \times 10^{23}$ átomos, de cada radioisótopo:

\begin{tabular}{|c|c|c|c|c|}
\hline Radioisótopo: & Meia-Vida (Anos): & Decaimento (MeV): & Atividade (Bq): & Potência (W): \\
\hline Hidrogenio-3 & 12,32 & 0,00186 & $1,07 \times 10^{15}$ & 3,19 \\
\hline Estrôncio-90 & 29,78 & 0,00546 & $4,44 \times 10^{14}$ & 38,82 \\
\hline Césio-137 & 30,07 & 0,00174 & $4,40 \times 10^{14}$ & 82,66 \\
\hline Actinio-227 & 21,77 & 0,00448 & $6,08 \times 10^{14}$ & 43,56 \\
\hline Cadmio-113m & 14,1 & 0,00316 & $9,39 \times 10^{15}$ & 47,45 \\
\hline Kriptõnio-85 & 10,17 & 0,00687 & $1,30 \times 10^{15}$ & 142,96 \\
\hline
\end{tabular}

"Escolhemos estudar ainda mais o Sr-90 como o material de origem com base em sua disponibilidade, mecanismo de decaimento e energias de emissão espectral. O Sr-90 é produzido como um produto de fissão em reatores nucleares e se acumula em quantidades adequadas em combustíveis nucleares. O Sr-90 concentra-se isotopicamente porque reside próximo a um pico n curva de rendimento da emissão e não requer purificação isotópica subseqüente na extração. No decaimento $\beta$, o Sr-90 decai em Y-90, que por sua vez sofre decaimento $\beta$ para produzir Zr-90 
estável" (Dixon, 2017). Conforme a afirmação, a utilização de elementos radioativos separados de restos nucleares, subprodutos da fissão nuclear, que se apresentam como um problema, pois atualmente, não há uma forma conhecida de reciclar ou reutilizar estes materiais e a melhor maneira no momento é estocar esses resíduos.

Quanto às técnicas de manufaturas, novos procedimentos deverão ser desenvolvidos, pois a engenharia necessária apresentará novos desafios, como a blindagem, necessária para que o gerador betavoltaico seja segura para o uso no cotidiano. Alguns elementos apresentam uma emissão de baixa energia de radiação beta, sem eventuais emissões gama, como é o caso do Hidrogênio-3 (H-3) e o Estrôncio 90 (Sr-90), que faz com que as blindagens relacionadas a esse radioisótopos se tornem mais seguras ao se comparar com outros, como o Criptônio 85 (Kr-85), que apresenta cerca de 99,63\% de emissão beta e cerca de 00,37\% de emissão secundária de raios gama durante seu decaimento.

Tabela 1. Relação entre a blindagem e os radioisótopos beta emissores.

\begin{tabular}{|c|c|c|}
\hline \multirow{2}{*}{ Radioisótopos: } & \multicolumn{2}{|c|}{ Espessura de Blindagem em: } \\
\cline { 2 - 3 } & Plástico $(\mathrm{cm})$ & Alumínio $(\mathrm{cm}):$ \\
\hline $\mathrm{H}-3$ (Trítio) & $0,001 \mathrm{~cm}$ & $<0,001 \mathrm{~cm}$ \\
\hline $\mathrm{Sr}-90$ & $1,1 \mathrm{~cm}$ & $0,52 \mathrm{~cm}$ \\
\hline $\mathrm{Ni}-63$ & $0,1 \mathrm{~cm}$ & $0,1 \mathrm{~cm}$ \\
\hline $\mathrm{Kr}-85$ & $0,25 \mathrm{~cm}$ & $0,21 \mathrm{~cm}$ \\
\hline
\end{tabular}

A radiação beta é conhecida por apresentar um elevado potencial de ionização e de penetração, considerada intermediária radiação alfa e gama. Entretanto, existem radioisótopos que apresentam emissão beta de menor energia, como o trítio, fazendo com que a blindagem de 
fontes de emissão beta pode ser realizada usando-se alguns centímetros de polímeros plásticos ou alguns milímetros de alumínio metálico, de acordo com as características dos radioisótopos, gerando uma blindagem segura.

Certos radioisótopos apresentam uma emissão de baixa energia, como ocorre com o trítio e o níquel 63 (Ni-63), onde as partículas beta não conseguem penetrar a camada de tecidos morto da pele, o que leva a algumas literaturas a desconsiderar blindagem nos casos.

Apesar do cálculo da blindagem tornar o gerador seguro, é importante lembrar que para um dispositivo portátil, avarias podem ocorrer e estimular falhas na blindagem, ocorridas durante o processo de fabricação, levando ao vazamento do conteúdo radioativo aos meio que os cerca, contaminando-o e oferecendo perigo às formas de vida próximas.

O cálculo de dose se faz pratico em caso de falhas de blindagem, pois permite calcular se a radiação emanada do gerador e a partir dessa informação, analisar com base nos limites de exposição estabelecidos pela Comissão Nacional de Energia Nuclear (CNEN).

Tabela 2. Dose de radioisótopos, em caso de falha e emissão total desta para o ambiente.

\begin{tabular}{|c|c|c|c|}
\hline Radioisótopos & Atividade (GBq) & Constante Gama $(\wp)$ & Dose por hora $(\mathbf{m S v} / \mathbf{h})$ \\
\hline $\mathrm{H}-3$ & $3,58 \times 10^{4}$ & - & - \\
\hline $\mathrm{Ni}-63$ & $4,41 \times 10^{3}$ & - & - \\
\hline Sr-90 & $1,48 \times 10^{4}$ & $6,52 \times 10^{-7}$ & 0,965776 \\
\hline Cd-113m & $3,13 \times 10^{4}$ & $3,75 \times 10^{-13}$ & $0,8603 \times 10^{-6}$ \\
\hline Kr-85 & $4,34 \times 10^{4}$ & $2,39 \times 10^{-7}$ & 0,9988 \\
\hline
\end{tabular}

A Tab.2 apresenta o cálculo de dos radionuclídeos mais práticos para atuar como base de um gerador betavoltaico. O cálculo de atividade foi baseado em cerca de 1/3 de material radioativo e o cenário apresentado como se toda a blindagem do gerador fosse perdida. 
Devido a baixa energia da emissão beta, como já descrito anteriormente, alguns radioisótopos dispensam uma blindagem mais significativa, pois suas emissão não tem energia suficiente para passar a camada de células mortas na superfície da pele humana, como é o caso do H-3 e do Ni-63.

Conforme o cálculo da Tab. 2, simulando uma vazamento total de radiação por falha da blindagem, sob o período de exposição constante de uma hora, a dose se aproxima de $1 \mathrm{mSv}$, valor próximo a dose anual recomendada pelo CNEN, Tab. 3.

Tabela 3. Tabela de limites de dosagens impostos pela CNEN.

\begin{tabular}{|c|c|c|c|}
\hline \multicolumn{4}{|c|}{ Limites de Dose Anuais } \\
\hline Grandeza & Órgão & $\begin{array}{c}\text { Individuo ocupacionalmente } \\
\text { exposto }\end{array}$ & Individuo do público \\
\hline Dose efetiva & Corpo inteiro & $20 \mathrm{mSv}$ & $1 \mathrm{mSv}$ \\
\hline \multirow{2}{*}{ Dose equivalente } & Cristalino & $20 \mathrm{mSv}$ & $15 \mathrm{mSv}$ \\
& Pele & (Alterado pela Resoluçăo CNEN 114/2011) & $50 \mathrm{mSv}$ \\
\cline { 2 - 4 } & Mãos e pés & $500 \mathrm{mSv}$ & -- \\
\cline { 2 - 4 } & & $500 \mathrm{mSv}$ & 5 \\
\hline
\end{tabular}

Entretanto, considerando o aspecto da produção em massa, com o uso em dispositivos de uso pessoal, como acontece com os atuais smartphones, se torna lógico pensar em situações onde a radiação beta "vaze", devido a avarias ou falhas no processo de confecção, levando a contaminação do ambiente e dos usuários.

Vazamentos acidentais podem ser impossíveis de serem evitados, entretanto, o dispositivo pode apresentar sistemas de diagnóstico, que alertara sobre falhas, relacionadas a problemas com o gerador. Se há vazamento na blindagem, um simples circuito de avaliação de potência gerada pode alertar quanto a vazamentos, pois caso a blindagem esteja comprometida, a potência gerada vai cair a um valor menor que o obtido na fabricação do mesmo, gerando um 
alerta e comunicação com a empresa que o fabricou, podendo chegar a outras autoridades responsáveis.

O uso preferencial de certos radioisótopos, como Trítio e Estrôncio-90, se deve a emissão radioativa predominante de partículas beta. Outros elementos podem apresentar emissões secundárias de radiação gama, como é o caso do criptônio 85 (Kr-85), onde 99,63\% de seu decaimento ocorre por emissão beta e cerca de $0,37 \%$ ocorre por emissão de raios gama. $\mathrm{O}$ produto do decaimento deve ser considerado, no caso, o Sr-90 apresenta um decaimento beta para o Y-90, um radioisótopo com meia-vida de 16hs, que decai através de decaimento gama, tornando-se esse isótopo contra indicado no uso de dispositivos portáteis.

Considerações Gerais. A utilização de geradores betavoltaicos em sistemas portáteis e demais aplicações relacionadas é uma opção viável e prática com a atual tecnologia, pois existem inúmeras aplicações em que há a necessidade de um funcionamento de longa duração.

"Os geradores de radioisótopos são uma das linhas relevantes do desenvolvimento de fontes de energia com um longo período de serviço desde a primeira vez que a possibilidade de obter energia elétrica durante o decaimento nuclear foi mencionada. A maioria desses geradores é projetada para fornecer energia a equipamentos eletrônicos de rádio, operar em objetos onde não há fontes de energia elétrica padrão ou onde há exigências rigorosas sobre a qualidade e continuidade do fornecimento de energia" (Bykov, 2017).

A eficiência do gerador é relativa a perda de potência em função do tempo, onde é possível se calcular a perda anual que um gerador betavoltaico pode apresentar em um ano, levando em consideração que sua potência inicial deverá ser maior que a do dispositivo, para garantir que depois de 05 anos, a alimentação se encontre em níveis aceitáveis para o funcionamento do dispositivo. Sistemas de regulagem de alimentação podem ser empregados para conter esse potencial inicial e regular a energia com o tempo para que o sistema eletrônico continue recebendo uma alimentação constante. 
Quanto ao aquecimento, geradores betavoltaicos podem aquecer mas não tem sua alimentação influenciada, pois as baterias convencionais têm uma perda considerável que ocorre entre a transformação de energia eletroquímica em energia elétrica, que oscila entre $85 \%$ nestes dispositivos, e tendo perdas em forma de energia térmica. No processo de um gerador de radioisótopos, a geração de energia em si é baseada em um processo não-térmico, o que ajuda no rendimento em si, chegando a um mínimo de 50\%, onde temos o fato do calor não participar do processo, facilitando a redução da escala dos geradores para aplicações em dispositivos móveis.

Um fator importante é a responsabilidade, as empresas que resolvam produzir geradores deste tipo, devem ter um comprometimento, onde seriam responsáveis pelo recolhimento no futuro e reciclagem destes produtos, reaproveitando materiais se possível. Um processo semelhante vem ocorrendo com as empresas que produzem veículos elétricos, onde elas já incorporam os preços de recolhimento e reciclagem das baterias usadas nestes veículos. Em termos de meio-ambiente, um radioisótopo é um elemento altamente nocivo para seres vivos, podendo causar alterações genéticas e até mesmo mortes, há casos famosos de acidentes radioativos em que vidas foram perdidas devido ao desconhecimento do fenômeno da radiação. Entretanto, mesmo se considerando que um elemento que decaia em radiação beta não seja um problema tão grave, é possível fazer com que o dispositivo que utilize esse gerador possa fazer seu próprio controle, através de algum algoritmo para o cálculo de vida útil dele, com base em sua perda de potência, ao passar dos anos ou caso ele apresente algum defeito. Considerando que o mesmo dispositivo tenha um funcionamento constante, é possível o uso de sistemas de localização, como o GPS, para se localizar estes geradores, caso seja perdido, possibilitando sua recuperação e evitando que a decomposição de seus materiais lance materiais radioativos em ecossistemas.

Geradores betavoltaicos são uma ótima alternativa às convencionais baterias em aplicações relativas, de forma a se apresentarem como alternativas as necessidades e não substituir totalmente, pois há aplicações que não necessitam de alguma qualidade adicional que 
uma bateria já não possua. A idéia de aproveitar energia de decaimento é antiga, presente em muitos projetos e em essência, é o mesmo princípio de empregar uma forma de energia e convertê-la para energia elétrica, no caso, uma energia fundamental.

Agradecimentos. Gabriel Salomão Ferreira agradece ao Instituto Federal de São Paulo (IFSP) e a todos os docentes envolvidos no Mestrado Acadêmico em Engenharia Mecânica.

\section{Referências}

(1) Alam, T. R., Spencer, M. G., Prelas, M. A., \& Pierson, M. A. (2018). Design and optimization of radioisotope sources for betavoltaic batteries. International Journal of Energy Research, 42(7).

(2) Bykov, A. S., Malinkovich, M. D., Kubasov, I. V., Kislyuk, A. M., Kiselev, D. A., Ksenich, S. V., ... Parkhomenko, Y. N. (2017). Application of Radioactive Isotopes for Beta-Voltaic Generators. Russian Microelectronics, 46(8), 527-539.

(3) Dixon, J., Rajan, A., Bohlemann, S., Coso, D., Upadhyaya, A. D., Rohatgi, A., ... Yee, S. (2016). Evaluation of a Silicon 90Sr Betavoltaic Power Source. Scientific Reports, 6(1).

(4) Rahastama, S., \& Waris, A. (2016). Analytical Study of 90Sr Betavoltaic Nuclear Battery Performance Based on p-n Junction Silicon. Journal of Physics: Conference Series, 739, 012003.

(5) Uhm, Y. R., Choi, B. G., Kim, J. B., Jeong, D.-H., \& Son, K. J. (2016). Study of a Betavoltaic Battery Using Electroplated Nickel-63 on Nickel Foil as a Power Source. Nuclear Engineering and Technology, 48(3), 773-777.

(6) G.R. Choppin, J.O.Liljenzin, J. Rydberg, "Radiochemistry and Nuclear Chemistry", 3rd edition, Butterworth-Heinemann, 2002. ISBN 978-0-7506-7463-8.

(7) Harder, D (1976), "[The new radiologic units of measurement gray and becquerel (author's translation from the German original)]", Röntgen-Blätter, 29 (1): 49-52, PMID 1251122.

(8) Basdevant, J.-L.; Rich, J.; Spiro, M. (2005). Fundamentals in Nuclear Physics: From Nuclear Structure to Cosmology. Springer. ISBN 978-0387016726.

(9) Kurie, F. N. D.; Richardson, J. R.; Paxton, H. C. (1936). "The Radiations Emitted from Artificially Produced Radioactive Substances. I. The Upper Limits and Shapes of the $\beta$-Ray Spectra from Several Elements". Physical Review. 49 (5): 368-381. Bibcode:1936PhRv...49..368K. doi:10.1103/PhysRev.49.368.

(10) Olsen, L.C. (December 1973). "Betavoltaic energy conversion". Energy Conversion. Elsevier Ltd. 13 (4): 117-124, IN1, 125-127. doi:10.1016/0013-7480(73)90010-7.

(11) Van Albada, G. D. et al. (2000). Measurement of mechanical vibrations excited in aluminum resonators by $0.6 \mathrm{GeV}$. Review of Scientific Instruments, 71 (3), 1345-1354. 